\title{
汉语词缀与构词 \\ CHINESE AFFIXES AND WORD FORMATION
}

\author{
Fu Ruomei \\ Chinese Departement, Faculty of Humanities, BINUS University \\ Jln. Kemanggisan Ilir III No.45, Kemanggisan - Palmerah, Jakarta 11480 \\ rosemary@binus.edu
}

\section{内容提要}

汉语属于典型的孤立语, 词类本身无形态标志, 附加成分少, 词根没有形态变化。汉语词汇的构成 方式以词根按照一定语法关系的组合为主。虽然词缀构词法不属于汉语构词的主流, 但词缀构词也是汉 语构词不可或缺的一部分。本文通过查阅文献资料, 总结汉语词缀类型、意义及构词功能。汉语类词缀 在构成新词上能力更强, 但汉语类词缀的发展方向还有待时间的考验。

关键词：汉语，词缀，类词缀，构词

\begin{abstract}
Chinese language is one of the typical isolated languages. It lacks morphological variation; part of speech has no morphological signs; the additional component of word formation is less; and the roots never change their forms. The major method of Chinese word formation is the combination of roots according to certain grammatical relations. Although the affix word formation is not part of mainstream Chinese word formation, affix-formation is still an integral part of the Chinese word-formation. Article used literature review, summarized the types and meanings of Chinese affixes. And meanwhile, article analyzed word formation function of Chinese Affixes and quasi-affixes. The Chinese quasi-affixes have stronger capabilities in forming new words, but development direction of Chinese quasi-affixes has to stand the test of time.
\end{abstract}

Keywords: Chinese, affixes, quasi-affixes, word formation 


\section{前言}

刘富华、孙炜在《语言学通论》（刘富华. 孙炜，2009：195）中指出：构词法指的是词构 成的方法, 是指构词成分（词根和词缀）通过不同方式组合成词的具体方法。语言中新词产生 的最主要方法是构词法, 它从结构形式的角度分析词的结构及新词产生的方法。词都是由一个 或几个语素构成的, 构词语素分两种, 一种叫词根, 指的是意义实在、在合成词内位置不固定 的成词语素和不成词语素; 一种叫词缀, 指的是意义不实在、在合成词内位置固定的不成词语 素。由一个语素构词的词, 叫做单纯词。由两个或两个以上的语素构成的词, 叫做合成词。合 成词有复合式、附加式、重叠式三种构词方式。附加式也叫加缀法, 是许多语言使用的最能产 的构词法之一。一个词根加上不同的词缀就构成了不同的新词。由于这些新词是在词根的基础 上产生出来的, 因而用这种方法产生的新词叫派生词。汉语附加式构词法虽然不是构词的主要 形式，但词缀在构词中也占有重要地位。

\section{内容}

《现代汉语知识大词典》（高更生, 1992：161）给出的词缀定义是：“词缀, 又称语 缀、词缀语素。指加在词根上面的构成新词的虚语素。词缀的作用是同词根构成合成词。一般 说来, 按照词缀同词根的位置关系, 可分为前缀、中缀、后缀三种。附加在词根前面的叫前 缀, 又叫词头、语头、前置成分。如 '老虎、阿妹、第五” 中的 '老、阿、第”。附加在词根 后面的叫后缀。如 ‘帽子、木头、姑娘家” 中的 ‘子、头、家”。加嵌在词根中间的叫中缀, 又称为词嵌。如 '对不起、吃得开” 中的 “不、得”。有的词缀单纯地表示语法意义; 有的既 表示一定的语法意义，又表示一定的词汇意义。有的词缀有表达感情和标志词性的作用。”

在汉语词缀的研究中, 近年来 “类词缀” 一词频繁出现。这一概念最早出现在《汉语语法 分析问题》（吕书湘，1978：48-49）。吕叔湘认为 “汉语里地道的语缀不很多, 前缀有 ‘阿”、“第”、“初”、“老”（鼠，虎，师，表）, “小’ (鸡儿, 孩儿, 辫儿）等, 后缀 有 ‘子’、“儿’、“头”、“巴’、“者”、“们”、“然”等，中缀只有 “得”、“不” （看得出, 看不出）。有不少语缀差不多可以算是前缀和后缀, 然而还是差点儿, 只可以称为 类前缀和类后缀。说它们作为前缀和后缀还差点儿, 还得加个 “类” 字, 是因为它们在语义上 还没有完全虚化, 有时候还以词根的面貌出现”。

词缀词汇意义模糊, 是词根的虚化, 构词时位置固定。汉语词缀构成新词能力弱, 现有带 词缀词多数不是新近产生的。类词缀在构成新词中更加活跃, 构成新词数量不断增加。类词缀 的构词能力强, 可以类推出很多相关的词。类词缀未来有两种可能的发展方向。一种可能是, 经过比较长时间的发展, 类词缀的词汇意义更加虚化, 逐渐成为词缀。另一种可能是词汇意义 仍旧保留，类词缀又回归为意义实在的词根。

\section{汉语词缀的类型}

词缀区别于词根的最主要特征是定位性和粘着性。现代汉语典型词缀共有 20 个, 其中前 缀 6 个, 后缀 11 个, 中缀 3 个。

前缀: 阿 初 第 老小打

后缀: 子家头儿们边巴尔乎然于

中缀: 里 不 得 
1. 前缀：阿 初 第 老 小 打

前缀 “阿” 多用于构成称呼, 用于排行、小名或姓前, 有亲昵的意味, 如: “阿六、 阿王、阿丽” 等; 用在某些亲属名称前, 如 “阿婆、阿妈、阿姨” ; “初” 加在数词前表 示阴历的日期, 如: “初一、初十” ; 前缀 “第” 构成序数词, 如: “第一、第二” ; “老” 语义虚化, 不再表示 “年纪大” 时, 可以用于称呼人、排行次序、某些动植物名, “老外、老二、老虎、老玉米” 等; 与 “老” 同理, “小” 在不表示 “年纪轻, 体积小” 时, 可作为词缀构成 “小姐、小丑、小心” 等; 前缀 “打” 语义已经虚化, 丧失了表示 “击打” 的意思, 可构成动词，如： “打的、打扫、打扮”。

2. 后缀: 子家头儿们边巴尔乎然于

后缀 “子” 是个名词后缀，也应读轻声，加在名词性词素后构成名词 “面子、帽子、 毯子、命根子”，加在形容词或动词性词素后，构成 “瘦子、矮子、梳子、掸子、垫 子”，也可构成数量词 “这档子（事）、一阵子、一下子、一伙子（人）”。“家”用在 某些名词后，表示属于那一类人，如：“女人家、孩子家、姑娘家、学生家”。这与类词 缀 “家”构成的 “歌唱家、艺术家” 不同，“女人家、孩子家” 中的“家” 意义已经虚 化，“歌唱家、艺术家” 中类词缀 “家” 仍带有 “某领域专家” 的含义。“头”作为后缀 应读轻声，可接于名词性词根后，构成名词 “舌头、骨头、罐头、苗头”，也可接在动词 性词根后, 构成 “想头、念头、饶头、看头” , 接在形容词词根后 “准头、苦头” 或作为 方位词后缀 “上头、里头、前头” 等。“儿” 多数情况作为名词后缀, 可构成名词 “眼 儿、盆儿、缝儿” 表示微小; 也可加在动词前将动词名词化 “盖儿、卷儿” ; 形容词名词 化 “亮儿、（蛋）黄儿、零碎儿” ; 具体事物抽象化 “门儿、根儿、油水儿” ; 区别不同 事物 “白面 白面儿（海洛因），老家 老家儿（父母和家中其他长辈）; 名词动词化 “火 火儿（他火儿了。表示 “生气”）”; 也可作为少数动词的后缀 “玩儿”。后缀 “们” 用于表示人称的复数, 如 “我们、他们、朋友们”。“边” 读儿化轻声时, 可作为 方位词后缀，构成方位词 “东边儿、里边儿、左边儿”；后缀 “巴” 可构成名词 “磕巴、 哑巴”，动词 “眨巴、嚼巴”，形容词 “咧巴、干巴、紧巴巴”; “尔” 形容词后缀, 构 成的形容词多作状语 “卓尔 (不群) 、率尔、莞尔 (而笑)” ; 也可构成副词 “偶尔”。

“乎” 充当动词后缀构成 “在乎、合乎 (规律) 、出乎 (预料)”，也可作为形容词或副 词后缀, 构成 “巍巍乎、郁郁乎、迥乎 (不同)、确乎（重要）”。“然” 可构成副词或 形容词 “忽然、突然、显然、猛然、飘飘然”，也可构成连词 “既然、虽然、纵然”。

“于” 可作为动词后缀, 构成 “合于、属于、在于、至于” 等, 也可作为形容词后缀构成

“勇于 (负责) 、善于 (调度) 、易于 (了解) 、难于 (实行) ”。

3. 中缀: 里 不 得

汉语中缀已经丧失了构词能力, 不再出现中缀构成的新词, 现有含中缀的词数量有 限, 主要有含中缀 “里” 的形容词 “稀里糊涂、土里土气、俊里俊气”; “不” 构成的

“对不起、吃不开、看不起”; “得” 构成的 “对得起、吃得开、看得起” 等。

4. 汉语类词缀主要有:

类前缀：半超 次大单多 反泛非负高 后 类 零

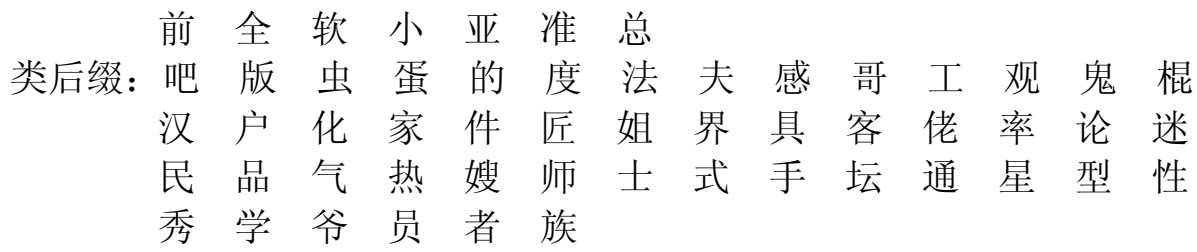

其中一些类词缀的形成是受西方语言影响, 用于表达西方语言中的一些词缀, 大部分这样 的类词缀还存在明显的词汇意义。如: 半 semi、超 super、次 sub-、单 mono-、微 micro-、 多 poly-/multi-、反 anti-/counter-、泛 pan-、非 non-/in-/un-、后 post-、前 pre-、亚 sub-、准 quasi-、化 ize-/-fy/-en/-tion、 
度-th/-ness/ity/-y/-sion/-ation/-ity、手-or/-er/-ant/-ary、性-ness/-ity/-ance/-y/ity、学-ies/-logy/-ism/-ing 等。

词缀在构造新词时具有一定的类化作用。类化性是汉语词缀的一个特点, 例如由后缀 “子”、“头”构成的都是名词，“打”、“于”则多构成动词。类词缀 “界”可以构成名词 “时尚界、体育界、艺术界、科学界、外交界等，表示某一特定领域。

\section{汉语词缀的意义}

词缀意义虚化的程度比较高, 理性义已经基本消失, 但只有少数是完全虚化的, 大多还存 有色彩义或感情义。词缀意义虚化、模糊, 但不代表完全没有意义。词缀是由词根虚化而来, 或多或少仍保存着一些词汇意义，“初” 在 “初一、初十” 中表示序列含义。“阿” 将“阿 姨”与母亲的姐妹 “姨” 的意义区分开来。“儿” 在 “鼻儿、窟窒儿、缝儿” 中都表示 “微 小”的含义。“汉语词缀有些可以标示词的类别，一般来说前缀“阿”、“老”，后缀 “子”、“家”、“头”构成的都是名词。“阿”、“老” 在构成对人的称呼时多表示亲 昵, 如 “阿三、老刘”。“打”、“于” 多构成动词。“乎”、“然” 都可以构成副词和形容 词，“乎”也可以构成动词 “在乎”。“巴” 可以构成名词 “亚巴”、动词 “眨巴” 和形容词 “干巴”。

相对于汉语词缀而言, 汉语类词缀数量庞大, 类词缀构词能力强, 还保留着一定的词汇意 义, 可以构成名词和区别词, 主要意思可以分类如下:
1. 表示某一类人:
(农) 家
(读) 者 (鼓) 手
(帅) 哥 (空) 姐
(舞) 星
(军) 嫂 (倒) 爷
(球) 迷
(木) 匠
(网) 虫
（上班）族 (笨) 蛋
(酒) 鬼
（奢）棍
(好) 汉
（阔）佬
（背包）客
（网）民
（医）师
(护) 士
（脚）夫
(电)工 (客)户
(职) 员

2. 表示某种性质: 半 (导体) 软 (饮料) 超（现实）次（大陆）

反 (倾销) 非 (正式) 负（增长） 类（词缀）

高（风险）小（报告）亚（健康）（普遍）性

3. 表示某种热潮：（汉语）热（出国）热

4. 表示某种感觉:（使命）感（亲切）感

5. 表示某一学科、理论: (人类) 学 (心理) 学 (相对) 论

6. 表示某一领域:（泳）坛（文艺）界

7. 表示场所，从 “bar酒吧” 类推而来: (茶) 吧 (网) 吧

8. 表示某种类型或方式: (轻) 型（节能）型（蝶）式 (台) 式

9. 表示时间: 前（清）后（现代）

10. 表示范围: 全 (天候) 全（自动）总（动员）

11. 表示尚未达到某种程度的: 准 (女婿) 准 (军事)

12. 表示某种交通工具：（面）的（摩）的

13. 表示某种表演:（时装）秀（真人）秀

14. 表示比率:（比）率、（效）率、（有效）率

15. 表示发生某种变化: (绿) 化 (净) 化 (数字) 化

16. 其他类词缀构成的名词: (盗) 版 (高) 度 (用) 法 (作) 品

（人生）观（硬）件（餐）具（一卡）通 


\section{汉语词缀在构词中的作用}

1. 古汉语单音节词的双音节化

在原单音词的前或后添加词缀构成双音词。

头: 石 $\rightarrow$ 石头 木 $\rightarrow$ 木头 前 $\rightarrow$ 前头

子: 桌 $\rightarrow$ 桌子 椅 $\rightarrow$ 椅子

老: 虎 $\rightarrow$ 老虎鼠 $\rightarrow$ 老鼠

2. 体现一定的模糊含义

词缀由词根虚化而来，尚存有一定的模糊含义，例如：“初一”、“第一”中的

“初”、“第”表示序列。

3. 标示词性

词缀 “子” 、类词缀 “度” 都是名词的标志, 而类词缀 “化” 则是动词的标志。某些汉 语词缀具有转变词类的功能, 动词 “盖、骗、梳”加词缀 “子”、“儿” 变成名词 “盖 子、盖儿、骗子、梳子”。

4. 为词根添加一定的色彩

汉语词缀具有比较浓郁的口语色彩，“木头、石头、盖儿” 都是非常口语化的。同时词 缀也可以体现感情色彩, 在人的姓或名前加 “阿” 表现出比较亲昵的感情, 如: “阿丽、 阿王”。

汉语中存在叠音后缀, 如: 红通通、绿油油、干巴巴、血淋淋、水汪汪、喜洋洋等。汉语 中还存在多重后缀, 所谓多重后缀是指多个后缀逐层加在词根上构成新词。例如: “孩子家” 中的 “子” 和 “家”; “石头子儿” 中的 “头”、“子”、“儿” 等。

\section{词缀的构词功能}

汉语词缀是词根在语言发展变化中逐渐虚化的结果，不属于汉语最初的语言成分。汉语词 缀曾有过很强的构词能力, “如在《现代汉语语法信息词典》中以 “儿” 结尾的词语有 176 个 ，其中 “儿” 作为词缀的有 157 个, 占 $89.2 \%$...”。（2011-

11：79）但词缀构词的高峰期已过, 基本不再产生新词, 相对而言类词缀在构成新词上的能力 更强。

汉语里中缀构成的词数量有限, 均已不再产生新词。汉语词缀可以叠加的很少, 仅限于一 些名词性后缀, 如: 小孩子家, 石头子儿等。书写形式上, 汉语词缀直接附加在词根上, 没有 任何书写变化。

汉语词缀主要构成名词、副词，少数可以构成动词（眨巴）、形容词（干巴、突然），后 缀 “然” 可以构成连词。汉语词缀不能构成介词。汉语词缀多为名词性词缀, 如 “子、头、家 、儿”。

汉语里同一词根与不同词缀结合构成不同的词的情况比较少见。词根与词缀的结合较为固 定。汉语中的词缀 “子、儿” 可以改变词类, 动词 “梳” 加词缀 “子” 变为名词, 形容词 “亮 ”加词缀 “儿” 变为名词, 汉语中缀 “里”

构成的形容词多表示贬义, 如 “稀里糊涂、土里土气、俊里俊气”。 


\section{结语}

汉语词缀存在前缀、后缀、中缀及类词缀。汉语词缀来自词根的虚化, 虽然理性义已经基 本消失，但大多还存有色彩义或感情义，只有少数是完全虚化的。词缀在构造新词时具有一定 的类化作用。汉语词缀主要构成名词、副词, 少数可以构成动词、形容词、连词, 但不能构成 介词。类词缀在构成新词上能力更强, 汉语以类词缀的形式对外来词缀进行吸收。汉语类词缀 能否进入词缀系统, 还有待时间的考验。在理解掌握汉语词缀时, 要注意具有相同书写形式的 词缀与词根的区分。汉语附加式构词词缀没有明显标志, 汉字 “老” 既可能是词缀, 如 “老 ( 鼠、虎）” 中的 “老”; 又可能是词根, 如 “老（人、妪）” 中的 “老”。我们不能认定 “老 ” 是一个词缀, 必须根据具体情况判断。判断一个构词成分在词中究竟是词缀还是词根, 必须 结合词的词汇意义。这也是掌握汉语词缀构词需要注意的一点。

\section{参考文献}

高更生. 现代汉语知识大词典. 济南:山东省教育出版社, 1992

黄伯荣, 廖序东. 现代汉语（增订四版）上、下册. 北京: 高等教育出版社, 2007

刘富华, 孙炜. 语言学通论. 北京: 北京语言大学出版社, 2009

刘中富. 实用汉语词汇. 合肥: 安徽教育出版社, 2003

吕叔湘. 汉语语法分析问题. 48-49 页. 北京:商务印书馆，1979

孙德金. 对外汉语词汇及词汇教学研究. 北京:商务印书馆, 2006

万艺玲. 汉语词汇教程. 北京: 北京语言大学出版社, 2000

万琴. 类词缀与词缀的共性特点分析. 温州职业技术学院学报, 2011, 第 11 卷（第 4 期） 